\title{
Rehabilitación cognitiva en trastornos psiquiátricos graves: aplicación de los subprogramas cognitivos de la terapia psicológica integrada (IPT).
}

\section{Cognitive rehabilitation in psychiatric serious disorders: application of the cognitive subprograms of the IPT.}

RESUMEN: El objetivo del estudio fue conocer los efectos de los subprogramas cognitivos de la Terapia Psicológica Integrada (IPT) en pacientes con trastornos psicóticos. La investigación, de tipo experimental, midió los cambios en los procesos cognitivos básicos y, a su vez, en la sintomatología, las habilidades sociales y de resolución de problemas. Los participantes fueron 32 pacientes en tratamiento ambulatorio diagnosticados de esquizofrenia y trastorno esquizoafectivo (F20 y F21), divididos en dos condiciones: experimental y control. Los participantes del grupo experimental asistieron a un programa de IPT durante seis meses, a razón de dos sesiones semanales. El grupo control no recibió ningún programa terapéutico de rehabilitación.

En el análisis de las medidas pre-post tratamiento para el grupo experimental, encontrándose una mejoría estadísticamente significativa tanto para la sintomatología cognitiva (Mpre=4,05 vs Mpost=3,60), como para la afectiva (Mpre=4,35 vs. Mpost=4,00) y la negativa (Mpre=4,65 vs Mpost=.4, 25). También, en las medidas del rendimiento se ha podido observar un incremento en el rendimiento (Mpre=12 vs. Mposts=13.95), en la medida de atención selectiva y memoria auditiva. Por último, se encontró una mejora en la satisfacción con la vida (Mpre $=55,60$ vs. Mpost= 53,67).

Los participantes del grupo control, por el contrario, no experimentaron cambios significativos en ninguna de las variables medidas.

Se evidencia la eficacia de la implementación de un programa de tratamiento psicológico integrado en la rehabilitación cognitiva de los pacientes psicóticos. Así mismo, se discuten las limitaciones y las implicaciones para futuras aplicaciones de la IPT en esta población.

PALABRAS CLAVE: Rehabilitación cognitiva, Terapia Psicológica Integrada, Esquizofrenia.
ABSTRACT: The aim of this study was know the effects of the cognitive subprograms of the IPT in patients with psychotic disorders. The experimental research measured the changes in the cognitive basic processes and, in turn, in symptomatology, social skills and problems resolution.

The participants were 32 patients in ambulatory treatment diagnosed of schizophrenia and schizoaffective disorder, divided in two conditions: experimental and control. The participants of the experimental group participated at IPT's program for six months, with the frequency of two weekly meetings. The group control did not receive any therapeutic program of rehabilitation.

The analysis of the measures pre-postest of the experimental group showed a statistically significant improvement in the cognitive symptomatology $(M=4,05$ vs $M=3,60)$, affective ( $M$ $=4,35$ vs. $=4,00)$ and denial $(M=5,00$ vs $M$ $=.4,75)$. As well as, in the cognitive performance ( $M=12$ vs. $M=13,95)$, mainly in selective attention and auditory report. Finally, we found an improvement in the satisfaction with the life $(M=55,60$ vs. $M=53,67)$. The participants of the control group did not experience significant changes in any of the measured variables.

The results demonstrate the efficiency of the implementation of a program of integrated psychological treatment to the cognitive rehabilitation of the psychotic patients. The limitations and the implications are discussed for future applications of the IPT in this population.

KEY WORDS: Cognitive rehabilitation, Integrated Psychological Therapy, Schizophrenia. 
ORIGINALES Y REVISIONES

\section{Introducción.}

Algunos de los campos más importantes de las intervenciones en los trastornos psicóticos están relacionados con las disfunciones cognitivas, identificadas por la Psicología de la Cognición como déficits perceptivos básicos o factores de vulnerabilidad, asociados con los trastornos psicóticos. Investigadores tales como Nuechterlein, Oltmanns y Neale, Asarnow y McCrimmon y Weinberger $(1,2,3,4)$ evidenciaron la presencia de deficiencias en el procesamiento de la información de los sujetos con trastorno psiquiátrico grave, concretándose estos desarreglos en los procesos de atención selectiva de memoria de trabajo y de vigilancia $(5,6,7)$.

El estudio de los déficits cognitivos en la esquizofrenia ha alcanzado gran relevancia, clarificándose las funciones cognitivas alteradas y su repercusión en la vida del sujeto. De esta manera se han identificado dos tipos de funciones que estarían alteradas en los pacientes que padecen psicosis; las funciones cognitivas básicas como las funciones ejecutivas, la memoria de trabajo, la atención, la capacidad de abstracción, la función motora y el lenguaje, por un lado, y las funciones de la cognición social, como la capacidad de preveer las reacciones de los demás, encontrar soluciones a los conflictos, percibir el entorno correctamente y planificar y resolver problemas por otro.

Se puede inferir que las alteraciones de las funciones cognitivas básicas serían las causantes de los problemas que presentan estos pacientes en las relaciones interpersonales y en la adaptación social, (8) así como uno de los factores que determinan la evolución del paciente con trastorno mental severo aunque es común utilizar el término severo, hay cierto acuerdo en que es más correcto el uso de Trastorno Mental Grave, el beneficio que éste pueda obtener de los programas de rehabilitación y la percepción subjetiva de su Calidad de Vida. Esto queda de manifiesto en el efecto que las disfunciones cognitivas pueden ejercer en cuestiones tales como el vivir de modo independiente, el establecimiento de relaciones interpersonales significativas, el logro de empleo o la adaptación a los cambios (9).

Recientemente se ha encontrado que las alteraciones cognitivas en los trastornos psicóticos resultan entidades independientes del trastorno, es decir, no parecen ser subproductos de los síntomas positivos o negativos de la enfermedad, ni del tratamiento con neurolépticos. En este sentido, autores como Nuechterlein y Dawson (11) Liberman y Green (5), Green y cols. (11) y Barrera (6), señalan que los déficits cognitivo-perceptivos serían factores de vulnerabilidad del sujeto para sufrir un trastorno psicótico, dependiendo de algunos factores como los señalados por el modelo de vulnerabilidad-estrés (12) y de los estresores psicosociales en el ambiente del sujeto. 
ORIGINALES Y REVISIONES

La vulnerabilidad premórbida cognitiva, por lo tanto, impediría la adquisición de habilidades sociales adaptativas, bloqueando la capacidad del paciente para afrontar los cambios producidos por la enfermedad o para resolver situaciones ambientales adversas. Brenner y cols. (13) sugieren que estos desarreglos hacen más vulnerable al sujeto, ya que reducen las estrategias de afrontamiento e interfieren en la adquisición de nuevos aprendizajes. Además, se ha encontrado que los desarreglos en las capacidades neurocognitivas juegan un papel importante en el ajuste comunitario del enfermo psicótico, vinculándose de forma específica y consistente con el desempeño del mismo en distintas áreas, incluyendo el desempeño social, funcional y ocupacional (14).

Los ámbitos de intervención cognitivo-conductual en las psicosis comprenden distintos déficits o anomalías (15), tanto los referidos a las funciones cognitivas básicas como a los déficits de procesamiento y gestión de estímulos sociales, pero siempre teniendo en cuenta que la presencia de déficits cognitivos básicos sería precursor o causa de alteraciones en las funciones de cognición social.

En resumen, estas intervenciones terapéuticas intervienen tanto en las disfunciones cognitivas como en las habilidades sociales, planteándose un enfoque de rehabilitación holístico del trastorno psicótico.

La Terapia Psicológica Integrada (IPT) de Brenner y colaboradores, es un programa terapéutico de corte cognitivo conductual consistente en una intervención jerarquizada por etapas. Su principal objetivo consiste en reducir los déficits cognitivos en las funciones de atención y percepción. Según los autores, las habilidades básicas como la concentración, la formación de conceptos, la capacidad de abstracción, las habilidades perceptivas y la memoria, deberían ser entrenadas en un primer momento para poder desarrollar luego formas más complejas de conducta social $(13,16)$. El modelo de Brenner y cols. ha sido diseñado para explicar los mecanismos que determinan y modifican la función cognitiva; así como para entender el efecto negativo de las alteraciones en el procesamiento de la información sobre otros aspectos del funcionamiento cotidiano, como puede ser la planificación o la competencia social $(17,8)$.

Brenner y colaboradores desarrollaron su programa de intervención basándose en el modelo de penetración $(13,16)$. La IPT está fundamentada en las diferencias que muestran los pacientes con esquizofrenia en los diferentes niveles de la organización de la conducta (nivel atencional-perceptivo; nivel cognitivo; nivel micro-social, y nivel macro-social). También establece que las deficiencias en un nivel pueden perjudicar el rendimiento en los otros niveles y que éstos guardan entre sí una disposición jerárquica; de esta manera, cambios en las funciones cognitivas básicas se asocian a cambios en funciones cognitivas más complejas, así como cambios de tipo funcional como la autonomía personal o el funcionamiento general (17). Es una terapia de intervención grupal con cinco 
subprogramas para la mejora de las habilidades deficitarias antes señaladas. Las primeras intervenciones van dirigidas a la rehabilitación de los procesos cognitivos y perceptivos, los programas intermedios transforman las habilidades cognitivas en respuestas verbales y sociales y las últimas intervenciones entrenan a los pacientes en el manejo y la resolución de los problemas interpersonales más complejos.

Los estudios realizados con IPT indican que el subprograma de Diferenciación Cognitiva mejora los procesos cognitivos elementales de los pacientes -aunque es cierto que el desempeño suele seguir por debajo del rango normal-, y que los efectos son visibles a corto y medio plazo (5). Cada subprograma se desarrolla de manera que, a medida que avanza la terapia se aumentan las exigencias al individuo, desde tareas simples y previsibles a otras más complejas. A la par, va decreciendo la estructuración de la terapia, finalizando con sesiones más flexibles y participativas.

El objetivo de este estudio fue encontrar un procedimiento terapéutico que evidenciara una mejoría de los déficits cognitivos en los pacientes que participaron en los subprogramas cognitivos de la IPT. Por otro lado, se esperó que, al mejorar en los procesos cognitivos básicos, las habilidades sociales y de resolución de problemas interpersonales se incrementara el rendimiento hacia un desempeño social más ajustado. Esta modificación fue valorada mediante escalas de ajuste social y satisfacción con la vida.

\section{METODO}

\section{Participantes}

Los participantes de este estudio fueron pacientes de los centros de Salud Mental (CSM) de Zumárraga y Beasain, con características sociodemográficas similares. Los criterios de selección de la muestra fueron el haber recibido el diagnóstico de esquizofrenia (F 20.) y de trastorno esquizoafectivo (F21.), según criterios CIE-10. El rango de edad fue de 24 a 64 años.

De acuerdo con estas características se seleccionaron inicialmente 35 pacientes, de los cuales 20 fueron asignados al grupo experimental y 12 al grupo control. Tres pacientes no cumplieron los criterios de inclusión y fueron desestimados.

El grupo experimental se subdividió, a su vez, en dos grupos de intervención clínica (con 12 y 8 miembros respectivamente). Esta asignación se realizó de manera intencionada, forzada por la ubicación geográfica de los participantes, que pertenecían a grupos terapéuticos previamente formados (Hospital de Día de 
ORIGINALES Y REVISIONES

Zumarraga y usuarios del taller ocupacional del CSM de Beasain). Todos los participantes de la condición experimental recibían una prestación económica por su grado de discapacidad psíquica. En el grupo control incluimos a varios sujetos que se encontraban activos laboralmente.

La mayoría de participantes convivían con su familia (72\%), el resto en un piso tutelado, solos o en pareja. El estado civil de la muestra indicó que la mayoría eran solteros $(84 \%)$. Cuatro de nuestros participantes tenían hijos.

Ambos grupos acudían a sus respectivos Centros de Salud Mental a recibir tratamiento psiquiátrico. Todos recibieron una sesión de información acerca del proceso terapéutico y firmaron un consentimiento informado en el que accedían a participar libremente en el estudio.

\section{Diseño}

Utilizamos un diseño cuasiexperimental de medidas repetidas para contrastar el efecto de la aplicación de la IPT. Se procuró igualar los grupos experimentales para controlar las posibles fuentes de error, con el fin de conseguir una buena validez para nuestro estudio. Para el posterior análisis de los datos se emplearon pruebas no paramétricas dado el tamaño muestral del estudio.

\section{Instrumentos}

Se administraron a ambos grupos las escalas que describiremos a continuación tanto al comienzo del tratamiento como a los seis meses, coincidiendo con la finalización del programa de intervención diseñado para el grupo experimental. El grupo control no recibe tratamiento IPT.

1. Instrumentos de medición para el Estado Clínico:

- Escala de Impresión Global de la Esquizofrenia (Clinical Global Impresión-Schizophrenia Scale, CGI-SCH). (18). Proporciona información cualitativa acerca de las principales dimensiones sintomatológicas del trastorno esquizofrénico (positiva, negativa, cognitiva, depresiva y global). Consta de dos subescalas, con cinco ítems cada una, que evalúan respectivamente, la gravedad del cuadro clínico y el grado de cambio. Se trata de una escala heteroaplicada, en el que el clínico debe puntuar cada uno de los ítems mediante una escala Likert de intensidad con los siguientes puntos de anclaje ( $1=$ normal, $7=$ gravemente enfermo). A pesar de ser ésta una medida cualitativa, presenta una buena validez convergente, con una correlación .43 ( $\mathrm{p}<.05)$ con el BPRS global en nuestra muestra.

-Brief Psychiatric Rating Scale (BPRS) (19). Proporciona información acerca de la sintomatología del paciente, enmarcándola en dos grandes grupos: síntomas positivos y negativos; además de dar un puntaje global. En nuestro trabajo hemos usado la versión de 18 ítems. Cada ítem se puntúa en una escala Likert de 5 grados de gravedad (1= ausencia del síntoma, 5= presencia en grado extremo).

2. Instrumentos de medición para el Perfil Cognitivo:

-WAIS-III Wechsler Adult Intellience intelligence Scale-Third Edition (20). 
Dada la longitud del instrumento, optamos por aplicar una versión reducida desarrollada especialmente por Blyer y su equipo (21) para la población con esquizofrenia. Ésta utiliza los subtest de información y aritmética de la escala verbal, y los subtest de cubos y clave de números, de la escala manipulativa. Mediante la corrección propuesta por el autor se obtiene un CI global que, ha sido comprobado empíricamente y correlaciona de manera significativa con los CI normativos del WAIS-III (21). De acuerdo con el objetivo de nuestro estudio, incluimos como medidas de cambio terapéutico la aplicación del subtest de dígitos junto con el subtest de aritmética, dada su relación empírica con las áreas entrenadas (ya que el subtest de dígitos evalúa la atención concentrada y la memoria auditiva inmediata; y el subtest de aritmética informa acerca de los niveles de compresión, pensamiento abstracto y asociativo).

- Mini Examen Cognoscitivo (MEC) (22). Se trata de un instrumento diseñado para la detección de casos de deterioro cognitivo. El paciente ha de contestar a 35 cuestiones que exploran las siguientes áreas: orientación temporal y espacial, concentración y cálculo, memoria diferida, lenguaje y praxis.

- Test de Fluencia Verbal (ST) (23). El propósito de este test es evaluar la producción espontánea de palabras dentro de una categoría preestablecida. Esta prueba mide el número de elementos de una categoría que puede evocar un sujeto en un tiempo determinado, habitualmente un minuto; se trata de una tarea compleja desde el punto de vista cognitivo, en la que intervienen procesos lingüísticos, mnésicos y ejecutivos.

3- Evaluación de la Adaptación social y la Satisfacción con la vida:

- Escala Autoaplicada de Adaptación Social (SASS) (24). Instrumento autoaplicado diseñado, en un primer momento, para evaluar la conducta y el ajuste social de los individuos con depresión y, posteriormente, ha sido utilizado ampliamente en otras patologías mentales. Consta de 21 items que exploran el funcionamiento del sujeto en cuatro áreas del funcionamiento comunitario: trabajo, familia, ocio y relaciones sociales. Cada item se valora en una escala Likert de rango 0 (peor funcionamiento) a 4 (mejor funcionamiento).

- Escala de Satisfacción con la Vida (Satisfaction with Life Domains ScaleSLDS) (25). Esta compuesta por 15 items de puntaje tipo Likert con rango 1 -muy insatisfecho- a 7 -muy satisfecho-) y proporciona información sobre cuatro dominios: seguridad, estimulación, pertenencia y realización, que son de especial relevancia en la percepción subjetiva de la Calidad de Vida. 
ORIGINALES Y REVISIONES

\section{Procedimiento}

El programa se desarrolló durante un período de seis meses, entre Marzo y Agosto de 2008, con una periodicidad de dos sesiones semanales de una hora de duración, por lo que el grupo experimental recibió un total de 50 sesiones. El esquema de sesiones seguido es similar al de otros estudios empíricos llevados a cabo con anterioridad (27). Las sesiones fueron guiadas por una terapeuta y una coterapeuta y se distribuyeron de la siguiente manera:

- Cuatro sesiones de psicoeducación, de una hora de duración durante las dos primeras semanas. Se trabajaron los siguientes contenidos: Esquizofrenia: curso, tipos y síntomas; tratamiento farmacológico; habilidades para la convivencia, y habilidades de comunicación.

- Diez sesiones del subprograma de Diferenciación Cognitiva, con una duración de cuarenta y cinco minutos. El objetivo de este subprograma es la mejora de las habilidades para focalizar y sostener la atención, para conceptualizar y generar estrategias de reconocimiento conceptual adecuadas, y la diferenciación de elementos esenciales y relativos.

- Diez sesiones del subprograma de Percepción Social, con una duración de treinta minutos; con el propósito de mejorar la percepción, la interpretación, la flexibilidad y el razonamiento de las situaciones sociales a la vez que se reducen los patrones desadaptativos.

- Diez sesiones del subprograma de Comunicación Verbal. La duración de este subprograma era de cuarenta y cinco minutos aproximadamente. Diseñado para incidir en la competencia de la escucha, la exactitud, competencia de la comprensión verbal y coherencia de la respuesta verbal. Las sesiones incluyeron: (a) Repetición literal de frases; (b) Repetición de frases según su sentido; (c) Preguntas autoformuladas con respuesta; (d) Interrogatorio acerca de un tema concreto; y (e) Comunicación libre.

- Diez sesiones del subprograma de Habilidades Sociales. Se realizó un entrenamiento en habilidades sociales, empezando por situaciones de bajo contenido emocional (inicio, mantenimiento y cierre de una conversación) hasta finalizar con situaciones con alta emotividad (hacer una reclamación). La técnica utilizada para la realización de este subprograma fue el juego de roles. Dada la técnica utilizada en este subprograma, la duración de estas sesiones era aproximadamente de una hora.

- Seis sesiones del subprograma de resolución de problemas interpersonales. Para identificar y modificar las conductas problemáticas específicas relacionadas con el ámbito interpersonal. En estas sesiones, se proponía al grupo una situación personal concreta para su discusión, siempre comenzando desde un contenido emocional neutro hasta situaciones más ansiógenas. El grupo, jun- 
to con el terapeuta y coterapeuta, revisaban los distintos puntos de vista y las conclusiones de cada miembro del grupo y, en consenso, se evaluaban todas las posibles vías de intervención, analizando los pros y contras para tras votación elegir la solución más apropiada para el conflicto. Posteriormente, se elaboraba entre todos un dialogo que era representado por los participantes. El último paso era el modelaje por parte del terapeuta, del role playing y el feedback a todos los participantes. En este subprograma se planteaba a los participantes que realizaran tareas para casa con el aquí y ahora del conflicto. Al igual que en el anterior subprograma, la duración de éste era de una hora.

\section{Resultados}

A la luz de los resultados obtenidos por otros grupos de investigación en distintas comunidades, así como en los distintos países donde se ha aplicado este enfoque terapéutico, era de esperar obtener una mejoría tanto en los niveles de autonomía del paciente como en el desempeño social (26).

Antes de comenzar con el análisis del efecto del tratamiento, se procedió a conocer las características iniciales de la muestra en cuanto a características sociodemográficas se refiere, aportándose datos de puntuaciones medias y frecuencias en las variables (tabla 1).

Con el objetivo de asegurar la homogeneidad de los participantes en el estudio se realizó un contraste no paramétrico para dos muestras no relacionadas de Mann Withney, para conocer las características de los participantes en relación a las variables de desempeño social y cognitivo, que luego serían tomadas como variables de mejora o cambio (tabla 2).

Los resultados muestran que ambos grupos, experimental y control, no presentaron características sociodemográficas ni de desempeño cognitivo o social significativamente diferentes. Así mismo, las características iniciales de los participantes no difieren significativamente, ni en desempeño intelectual (medido con el WAIS-III) ni en la mediada de deterioro cognitivo (medida con el MEC), ni en la medida de ajuste social (evaluada mediante la SASS), ni en la percepción subjetiva de la calidad de vida (registrada mediante la SLDS).

Se procedió del mismo modo para el análisis de las medidas clínicas, encontrándose que tampoco diferían las puntuaciones de psicopatología (medida con las escalas BPRS y CGI-SCH) de ambos grupos al inicio del tratamiento. Sin embargo, se encontraron diferencias estadísticamente significativas en la gravedad de la sintomatología positiva medida con la escala CGI-SCH, presentando el grupo de control niveles mayores que el grupo experimental de sintomatología 
ORIGINALES Y REVISIONES

productiva, a pesar de esto, las diferencias no presentaron ningún problema a la hora de comparar los grupos (tabla 3 ).

Con el fin de estudiar la relación del efecto de los subprogramas terapéuticos sobre la psicopatología de los sujetos, el desempeño cognitivo y la satisfacción con la vida, se aplicó a cada grupo una prueba no paramétrica de dos muestras relacionadas de Wilcoxon, midiendo las variables antes del inicio del tratamiento (pretest) y una vez concluido (postest). Este análisis nos permite comprobar el tamaño del efecto entre ambos grupos. Todos los contrastes realizados para ambos grupos aparecen reflejados en la tabla 4.

En relación a las medidas clínicas, se encontraron diferencias estadísticamente significativas en CGI global $(Z=-2.24$; $p<.05)$; CGI negativo $(Z=-2.82$; $\mathrm{p}<0.5)$; CGI cognitivo $(\mathrm{Z}=-3.0 ; \mathrm{p}<.05)$; CGI depresivo $(\mathrm{Z}=-2.33 ; \mathrm{p}<.05)$ mejorando por consiguiente todos estos aspectos. En los síntomas productivos (CGI positivos) no hubo diferencias pre-post tratamiento. En el grupo control no se encontraron diferencias estadísticamente significativas para ninguno de los indicadores de gravedad sintomática de la enfermedad.

Los hallazgos más consistentes indican, por lo tanto, que existen beneficios del tratamiento psicoterapéutico en la reducción de la gravedad de la sintomatología clínica. Los efectos del programa terapéutico encontrados, medidos con los subtest del WAIS-III de dígitos y aritmética, ponen de manifiesto mejorías significativas en el primero de los indicadores $(Z$ dígitos $=-2.06 ; \mathrm{p}<.05)$ pero no en aritmética, como se puede observar en la tabla 4.

Así mismo, en el análisis de la Satisfacción con la Vida (Z SLDS= -3.04; $\mathrm{p}<.05)$ indica que el grupo experimental presenta mejorías al final del tratamiento en relación a la satisfacción con la vida percibida por el equipo clínico. Estas puntuaciones están reflejadas en la tabla anterior.

En resumen, los resultados de las diferencias de medias pre-post tratamiento, según grupo, muestran la eficacia de la terapia en los participantes del grupo experimental, tanto en las medidas de sintomatología como del déficit cognitivo. Sin embargo, esto no ocurre con el grupo de control, ya que no se encontraron diferencias estadísticamente significativas en ninguna de las variables del grupo de control.

\section{Discusión}

Los resultados que hemos encontrado en el estudio apoyan la idea de que la IPT es eficaz para disminuir los síntomas clínicos y déficits cognitivos inherentes al trastorno esquizofrénico, mejorando la calidad de vida de este tipo de pacien- 
tes. Esto corrobora lo encontrado en otros estudios empíricos como los de Roder y Brenner (16), Vallina y Lemos $(27,26)$ y Zimmerman y colaboradores (28). En estos se defienden los beneficios que un tratamiento integral multimodal biopsicosocial obtiene en comparación con los tratamientos basados únicamente en un aspecto del trastorno.

De la misma manera, los autores originales establecen que la mejora de la cognición en la esquizofrenia es la estrategia más importante en los tratamientos integrales de esta enfermedad. Los datos obtenidos ponen de manifiesto que el programa de IPT reduce las alteraciones cognitivas básicas, disminuyendo de manera global la sintomatología clínica y mejorando las habilidades sociales de los pacientes participantes del programa (29).

En nuestro proyecto esperábamos que los cambios experimentados en la sintomatología depresiva, cognitiva y negativa, ayudarían a los pacientes en su socialización y en la capacidad de resolución de problemas cotidianos, dado que es esperable que una persona con altas puntuaciones en estos aspectos presente peores índices de socialización, apatía a la hora de establecer relaciones, desmotivación y pautas sociales poco adaptativas.

Podemos confirmar que nuestras hipótesis se han cumplido, particularmente en relación a las medidas de sintomatología y de procesos cognitivos básicos. Por lo que se refiere a la relación con las medidas de desempeño social y de resolución de problemas, el cambio que se ha producido no ha ido en la dirección esperada.

Ello podría explicarse atendiendo a que el cambio en los procesos sociorelacionales suele ser más sutil, lento y menos estable a través del tiempo. Cabe destacar que nuestros resultados coinciden con los hallazgos de otros autores quienes señalan que el impacto de la IPT se refleja principalmente en los procesos cognitivos $(13,30,31)$. Además este cambio es evidenciable a corto plazo, siendo necesario realizar estudios de seguimiento para establecer conclusiones firmes sobre los procesos sociales.

Finalmente, cabe señalar que en la aplicación del programa nos hemos encontrado con las dificultades clínicas características de estas enfermedades. La mayoría de los sujetos presentaban, al principio del programa, dificultades para participar en conversaciones grupales o se presentaban apáticos y desmotivados, sobre todo los más deficitarios. Otros, por el contrario, estaban demasiado ansiosos y monopolizaban las sesiones. Por esta razón, nos propusimos crear en los grupos de terapia grupal normas que los participantes se comprometieron a seguir (asistencia, respeto de turnos al hablar, participación en las tareas, motivar compañeros...). Esto, junto con la adaptación de los materiales de los subprogramas a la realidad concreta de los participantes, permitió que conformáramos grupos de terapia sólidos y participativos, con una buena implicación. 
ORIGINALES Y REVISIONES

\section{Aplicación Clínica}

Podemos concluir que la aplicación del programa de IPT realizado ha resultado eficaz. No obstante, investigaciones recientes apoyan la idea de incorporar a la familia para una mejora de los resultados. Estos trabajos mostraron un aporte a la mejoría obtenida por la IPT al instruir a las familias en el conocimiento de la enfermedad, déficits, tratamiento farmacológico, efectos adversos, reconocimiento de pródomos, etc., así como el entrenamiento en el manejo de la emoción expresada, destinada a reducir la influencia de estados emocionales perturbadores en el funcionamiento cognitivo y social $(17,26,27,29,28,31)$.

Otra de las circunstancias que aumentaría la eficacia de la IPT sería la aplicación repetida del programa y con una duración superior a seis meses. Por la naturaleza misma del desarrollo de la patología cognitiva, está claro que una duración superior de la terapia resultaría más efectiva, así como la repetición de este entrenamiento, con el objetivo de que los pacientes logren mantener la ganancia de las habilidades obtenidas.

Concluyendo, podemos afirmar que la aplicación de un tratamiento psicológico integrado en los trastornos psicóticos ha resultado eficaz para la rehabilitación de pacientes crónicos de nuestro sistema sanitario. Sobre todo, en la reducción de los déficits cognitivos básicos, la sintomatología clínica y la resolución de problemas a corto plazo.

Recomendamos, finalmente, tener en cuenta a la familia en el proceso de rehabilitación; así como, la realización de estudios de seguimiento para comprobar la eficacia de los resultados a corto y medio plazo.

Tabla 1

Características sociodemográficas de la muestra.

\begin{tabular}{l|c|c|}
\hline & GRUPO & GRUPO \\
& CXPERIMENTAL & 12 \\
\hline Numero sujetos & 20 & 42.0 (de 29 a 53) \\
\hline Edad (media y rango) & 44.2 de 24 a 64$)$ & $25 \%$ mujeres, \\
\hline Sexo (\%) & $30 \%$ mujeres; & $75 \%$ hombres \\
\hline Estado civil (\%) & $70 \%$ hombres & $75 \%$ solteros \\
& $75 \%$ solteros & $16 \%$ casados \\
& $15 \%$ casados & $0,84 \%$ viudos \\
& $5 \%$ viudos & $58 \%$ activos \\
& $5 \%$ divorciados & 1 laboralmente \\
\hline Estado laboral (\%) & $80 \%$ parados/incapacitados & $42 \%$ parados/discapacitados \\
& 1 laboralmente. & $33,3 \%$ mas 20 años \\
& $20 \%$ activos & $33,3 \%$ entre 16 y 20 años \\
\hline
\end{tabular}


Tabla 2

Medidas de desempeño social y cognitivo al inicio del tratamiento $(n=32)$

\begin{tabular}{|c|c|c|c|c|}
\hline & \multicolumn{2}{|c|}{$\begin{array}{c}\text { GRUPO } \\
\text { EXPERIMENTAL }\end{array}$} & \multicolumn{2}{|c|}{$\begin{array}{c}\text { GRUPO } \\
\text { CONTROL }\end{array}$} \\
\hline & Media & d.t. & Media & d.t. \\
\hline SLDS & 50,70 & 8,11 & 51 & 7,66 \\
\hline SASS & 37,35 & 12,71 & 42,58 & 13,95 \\
\hline $\mathrm{MEC}$ & 26,65 & 2.87 & 26,67 & 2,87 \\
\hline WAIS III $_{\text {TOTAL }}$ & 84,10 & 21,68 & 86,50 & 16,71 \\
\hline SET TEST & 35,95 & 5,18 & 36,92 & 2,99 \\
\hline
\end{tabular}

SLDS: Satisfaction With Life Domains Scale. (Baker \& Intagliata).

SASS: Social Adaptation Self-Scale. (Bosc, Dubini \& Polin).

MEC: Mini Examen Cognoscitivo (Lobo).

WAIS III: Wechsler Adult Intelligence Scale Third Edition (Wechsler).

SET TEST: Test de Fluencia Verbal Categorial.

Tabla 3

Medidas clínicas al inicio del tratamiento.

\begin{tabular}{|c|c|c|c|c|}
\hline & EXPF & TAL & & \\
\hline & Media & d.t. & Media & d.t. \\
\hline$B P R S_{\text {positivo }}$ & 5,50 & 3,23 & 5,92 & 3,23 \\
\hline$B P R S_{\text {negativo }}$ & 6,30 & 3.097 & 7,83 & 2,44 \\
\hline$B P R S_{\text {total }}$ & 2,55 & .759 & 2,67 & .492 \\
\hline$C G I_{\text {gravedad positivo }}$ & 4,20 & 1,28 & 4,92 & .669 \\
\hline$C G I_{\text {gravedad negativo }}$ & 4,65 & 1,22 & 4,92 & 1,16 \\
\hline$C G I_{\text {gravedad afectivo }}$ & 4,35 & 1,50 & 4,92 & 1,37 \\
\hline$C G I_{\text {gravedad cognitivo }}$ & 4,05 & 1,50 & 3,67 & 1,49 \\
\hline$C G I_{\text {gravedad global }}$ & 5 & .649 & 5,08 & .669 \\
\hline
\end{tabular}

BPRS: Brief Psychiatric Rating Scale. (Overall \& Gorham).

CGI-SCH: Clinical Global Impression for Schizophrenia (Haro et al.) 
ORIGINALES Y REVISIONES

Tabla 4

Resultados de la prueba de los rangos por signo de Wilcoxon para las variables sintomatología, desempeño cognitivo y satisfacción con la vida $(n=32)$. (Medidas pre y post tratamiento)

\begin{tabular}{|c|c|c|c|c|c|}
\hline Variables & Grupo & $\begin{array}{c}\text { Media } \\
\text { Pre post }\end{array}$ & \begin{tabular}{|c|} 
Desviación típica \\
Pre post
\end{tabular} & $\begin{array}{c}\text { Puntuación } \\
\text { Z }\end{array}$ & \begin{tabular}{|c|} 
Significación \\
asistólica bilateral
\end{tabular} \\
\hline $\begin{array}{l}\text { Sintomatologia } \\
\text { negativa }\end{array}$ & $\begin{array}{l}\text { Experimental } \\
\text { Control }\end{array}$ & $\begin{array}{l}4,65-4,25 \\
4,92-4,83\end{array}$ & $\begin{array}{l}1,22-1,11 \\
1,16-1,11\end{array}$ & $\begin{array}{l}-2,82 \\
-1,00\end{array}$ & $\begin{array}{l}.005 \\
.317\end{array}$ \\
\hline $\begin{array}{l}\text { Sintomatología } \\
\text { cognitiva }\end{array}$ & $\begin{array}{l}\text { Experimental } \\
\text { Control }\end{array}$ & $\begin{array}{l}4,05-3,60 \\
3,67-3,75\end{array}$ & $\begin{array}{c}.74-1,31 \\
1,49-1,42\end{array}$ & $\begin{array}{l}-2,33 \\
-.577\end{array}$ & $\begin{array}{l}.020 \\
.564\end{array}$ \\
\hline $\begin{array}{l}\text { Sintomatología } \\
\text { afectiva }\end{array}$ & $\begin{array}{l}\text { Experimental } \\
\text { Control }\end{array}$ & $\begin{array}{l}4,35-4,00 \\
4,92-4,83\end{array}$ & $\begin{array}{r}.745-.725 \\
1,37-1,26\end{array}$ & $\begin{array}{l}-3.00 \\
-1,00\end{array}$ & $\begin{array}{l}.003 \\
.317\end{array}$ \\
\hline $\begin{array}{l}\text { Sintomatología } \\
\text { global }\end{array}$ & $\begin{array}{l}\text { Experimental } \\
\text { Control }\end{array}$ & $\begin{array}{l}5,00-4,75 \\
5,08-4,92\end{array}$ & $\begin{array}{l}.649-.639 \\
.669-.515\end{array}$ & $\begin{array}{l}-2,236 \\
-1,14\end{array}$ & $\begin{array}{l}.025 \\
.157\end{array}$ \\
\hline Dígitos & $\begin{array}{l}\text { Experimental } \\
\text { Control }\end{array}$ & $\begin{array}{c}12-13,95 \\
11,08-9,58\end{array}$ & $\begin{array}{l}5,15-3,73 \\
4,75-5,94\end{array}$ & $\begin{array}{l}-2,06 \\
-.878\end{array}$ & $\begin{array}{l}. \mathbf{0 3 9} \\
.413\end{array}$ \\
\hline Aritmética & $\begin{array}{l}\text { Experimental } \\
\text { Control }\end{array}$ & $\begin{array}{l}7,79-6,55 \\
6,75-6,67 \\
\end{array}$ & $\begin{array}{l}3,98-4,16 \\
3,62-4,36 \\
\end{array}$ & $\begin{array}{l}-1,38 \\
-.878\end{array}$ & $\begin{array}{l}.168 \\
.380\end{array}$ \\
\hline$\overline{S L D S}$ & $\begin{array}{l}\text { Experimental } \\
\text { Control }\end{array}$ & $\begin{array}{c}50,70-55,60 \\
51-53,67\end{array}$ & $\begin{array}{l}8,11-6,40 \\
7,66-5,80\end{array}$ & $\begin{array}{l}-3,036 \\
-1,79\end{array}$ & $\begin{array}{l}.002 \\
.073\end{array}$ \\
\hline
\end{tabular}

\section{BIBLIOGRAFÍA:}

(1) NuECHTERLEIN KH. Refocusing on attentional dysfunctions in schizophrenia. Schizophrenia Bulletin. 1977; 3: 457-469.

(2) Oltmanns TF \& Neale JM. Schizophrenic performance when distractors are present: Attentional deficit or differential task difficulty? Journal of Abnormal Psychology. 1975; 84: 205-209.

(3) Asarnow RF; MaCCRimmon DJ. Residual performance deficit in clinically remitted schizophrenics: A marker of schizophrenia? Journal of Abnormal Psychology. 1978; 87. 597-608.

(4) WeinBerger DR; Berman KF \& Zec RZ. Physiologic dysfunctions of dorsolateral prefrontal cortex in schizophrenia: I. Regional cerebral blood flow evidence. Archives of General Psychiatry. 1986; 43:114-124.

(5) Libermann RP; Green MF. Whither Cognitive Behavioural Therapy for schizophrenia? Schizoprhenia Bulletin. 1992; 18 (Suppl 1): 27-35.

(6) BARRERA PA. Los trastornos cognitivos en la esquizofrenia. Revista chilena de neuropsiquiatria. 1992; 44 (Suppl 3): 215-221.

(7) De la Higuera J; Sagastagortia E. Rehabilitación cognitiva en la esquizofrenia: estado actual y perspectivas futuras. Apuntes de Psicología. 2006; 24 (Suppl 1-3): 245-266.

(8) Bousoño M. La esquizofrenia y sus dimensiones.Euromedia Editores.2009.

(9) Wilder-Willis KE, Shear PK; StefFen J Y Borkin J. The relationship between cognitive dysfunctionmand coping abilities in schizophrenia. Schizophrenia Research. 2002; 55: 259-267. 
ORIGINALES Y REVISIONES

(10) NuECHTERLEIN KH \& DAwson ME. Information processing and attentional functioning in the developmental course of schizophrenic disorders. Schizophrenia Bulletin. 1984; 10: 160-204.

(11) Green MF; Kern R; BrafF D \& Mintz D. Neurocognitive deficits and Functional outcome in Schizophrenia: Are we measuring the "right stuff"? Schizophrenia Bulletin. 2000; 1 (Suppl 26): 119-136.

(12) ZuBIN J \& SPRING B. Vulnerability: a new view of schizophrenia. Journal of Abnormal Psychology. 1977; 86 (Suppl 2): 103-126.

(13) Brenner HD; Hodel B; Roder V \& Corrigan P. Dysfunctions and Behavioural Deficits in Schizophrenia. Schizophrenia Bulletin. 1992; 18 (Suppl 1): 21-25.

(14) Cohen AS; Forbes CB; Mann MC \& Blanchard JJ. Specific cognitive deficits and different domains of social functioning impairment in schizophrenia. Schizophrenia Research. 2006; 81 (Suppl 2-3): 227-238.

(15) Davidson L; LAmbert S; \& McGlashan TC. Psychoterapeutic and cognitive-behavioural treatments for schizophrenia: Developing a disorder-specific form of psychotherapy for persons with psychosis. In C. Perris \& PD McGorry (Eds.). Cognitive psychotherapy of psychotic and personality disorders: Handbook of theory and practice. New York Wiley.1998; p. 1-20.

(16) Roder V; Brenner HD; Hodel B \& Kienzle N. Terapia Integrada de la esquizofrenia. Barcelona: Ariel Psicología.1996.

(17) Hodel B; BrenNer HD. Cognitive therapy with schizophrenic patients: conceptual basis, present state, future directions. Acta Psychiatrica Scandinavica. 1994; 90: 108-15.

(18) Haro JM; Kamath SA; Movick D, ET AL. The Clinical Global Impression-Schizophrenia Scale: a simple instrument to measure the diversity of symptoms presents in schizophrenia. Acta Psychiatrica Scandinavica. 2003; (Suppl. 46): 16-23.

(19) Overall JE \& Gorham DR. The Brief Psychiatric Rating Scale. Psychological Report 1962; 10: 799-812.

(20) WechsLer, D. Test de inteligencia para adultos: WAIS-III. Manual técnico. Buenos Aires, Paidos. 2002.

(21) Blyer, CR; Gold, JM; IAnnone, VN \& Buchanan, RW. Short form of the WAIS-III for use with patients with schizophrenia. Schizophrenia Research. . 2000; 46: 209-215.

(22) Lово A. Mini-Examen Cognoscitivo: Versión española del Mini-Mental Status Examination de Folstein et al (1975).Actas Luso-Españolas de Neurología, psiquiatría y ciencias afines. 1979; 3: 189-202.

(23) Isaacs B, Akhtar AJ. The set Test: a rapid test of mental function in old people. Age and Ageing. 1972: 222-6.

(24) Bosc M; Dubini A \& Polin V. Development and validation of social functioning scale: the Social Adaptation Self-evaluated Scale. European Neuropsychopharmacology 1997; 7 (Suppl 1): 57-70.

(25) Baker F, Intagliata J. SLDS Satisfaction with Life Domains Scale .Quality of life in the evaluation of community support systems. Eval Program Plann. 1982; 5: 69-79.

(26) Vallina Fernández O \& Lemos Giraldez S. Tratamientos eficaces para la esquizofrenia. Psicothema. 2001; 13 (Supp 13): 345-364.

(27) Valuina Fernández O; Lemos Giraldez S; Alonso Sánchez M \& Gutiérrez Perez A.M. Tratamiento Psicológico Integrado de pacientes esquizofrénicos. Psicothema. 1998; 10 ( Suppl 2): 459-474.

(28) Zimmer M; Veríssimo DA; Laitano D; Ferreira EE \& Belmonte de Abreu P. A twelveweek randomized controlled study of the cognitive-behavioural Intergrated Psychological Therapy program: positive effect on the social functioning of chizophrenic patients. Revista Brasileña de Psiquiatria. 
2007; 29 (Suppl 2): 140-147.

(29) Vallina Hernández O; Lemos Giraldez S; Roder V; García Sanz A; Otero García A et AL. Rehab rounds: An Integrated Treatment Program for Schizophrenia. Psychiatric Services. 2001; 52: 1165-1167.

(30) Roder V; Mueller DR; Mueser KT \& Brenner HD. Integrated Psychological Therapy (IPT) for Schizophrenia: Is it effective? Schizophrenia Bulletin. 2006; 32: 81-93.

(31) Spaulding WD; Reed D; Sullivan M; Richardson C \& Weiler M. Effects of cognitive treatment in psychiatric rehabilitation. Schizophrenia Bulletin. 1999; 25 (Supl 4): 657-676.

* GABALDÓN POC, O. Dra. en Psicología. Psicóloga Clínica. * RUIZ IRIONDO, M. Psicóloga. Becaria de Investigación.* BILBAO RAMÍREZ, Ma A. Dra. en Psicologia. Psicóloga de la Salud. ** BURGUERA, J.M ${ }^{\mathrm{a}}$. Terapeuta Ocupacional**** ALÚSTIZA GARCÍA, A. Enfermera Psiquiátrica* ERKIZIA AMILIBIA, B. Médico Psiquiatra* SAKARA MAGALLÓN, Mª J. Médico Psiquiatra ***

Facultad de Psicología - Universidad del País Vasco. **.

Salud Mental Extrahospitalaria de Gipuzkoa (CSM-Beasain * y CSM Zumárraga***)

Correspondencia: Ciudad Jardín, nº 20 -20700 Zumarraga. Telf: 943721563 / 63572555

Correo electrónico: Olga.gabaldonpoc@osakidetza.net

Agradecimientos: Este estudio ha sido financiado dentro del Contrato Programa por el Departamento de Sanidad del Gobierno Vasco, resolución - No 131/2008. Los autores quieren agradecer al Dr. Álvaro Iruin por su contribución y apoyo en la realización de éste artículo.

** Recibido: 29/06/2009 